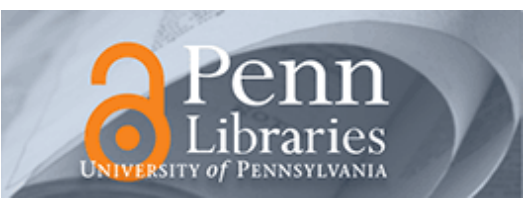

University of Pennsylvania

ScholarlyCommons

\title{
AKA-TPG: A Program for Kinetic and Epidemiological Analysis of Data from Labeled Glucose Investigations Using the Two-Pool Model and Database Technology
}

\author{
Raymond C. Boston \\ Darko Stefanovski \\ University of Pennsylvania, sdarko@vet.upenn.edu \\ Jan E. Henriksen
}

Glen M. Ward

Peter J. Moate

Follow this and additional works at: https://repository.upenn.edu/vet_papers

Part of the Medicine and Health Sciences Commons

\section{Recommended Citation}

Boston, R. C., Stefanovski, D., Henriksen, J. E., Ward, G. M., \& Moate, P. J. (2007). AKA-TPG: A Program for Kinetic and Epidemiological Analysis of Data from Labeled Glucose Investigations Using the Two-Pool Model and Database Technology. Diabetes Technology \& Therapeutics, 9 (1), 99-108. http://dx.doi.org/ 10.1089/dia.2006.0044

At the time of publication, author Darko Stefanovski was affiliated with the University of Southern California. Currently, he is a faculty member at the University of Pennsylvania's School of Veterinary Medicine.

This paper is posted at ScholarlyCommons. https://repository.upenn.edu/vet_papers/143

For more information, please contact repository@pobox.upenn.edu. 


\title{
AKA-TPG: A Program for Kinetic and Epidemiological Analysis of Data from Labeled Glucose Investigations Using the Two-Pool Model and Database Technology
}

\begin{abstract}
Background: The Two-Pool Glucose (TPG) model has an important role to play in diabetes research since it enables analysis of data obtained from the frequently sampled labeled (hot) glucose tolerance test (FSHGT). TPG modeling allows determination of the separate effects of insulin on the disposal of glucose and on the hepatic production of glucose. It therefore provides a basis for the accurate estimation of glucose effectiveness, insulin sensitivity, and the profile of the rate of endogenous glucose production. Until now, there has been no program available dedicated to the TPG model, and a number of technical reasons have deterred researchers from performing TPG analysis.
\end{abstract}

Methods and Results: In this paper, we describe AKA-TPG, a new program that combines automatic kinetic analysis of the TPG model data with database technologies. AKA-TPG enables researchers who have no expertise in modeling to quickly fit the TPG model to individual FSHGT data sets consisting of plasma concentrations of unlabeled glucose, labeled glucose, and insulin. Most importantly, because the entire process is automated, parameters are almost always identified, and parameter estimates are accurate and reproducible. AKA-TPG enables the demographic data of hundreds of individual subjects, their individual unlabeled and labeled glucose and insulin data, and each subject's parameters and indices derived from AKA-TPG to be securely stored in, and retrieved from, a database. We describe how the stratification and population analysis tools in AKA-TPG are used and present population estimates of TPG model parameters for young, healthy (without diabetes) Nordic men.

Conclusion: Researchers now have a practical tool to enable kinetic and epidemiological analysis of TPG data sets.

Disciplines

Medicine and Health Sciences

\section{Comments}

At the time of publication, author Darko Stefanovski was affiliated with the University of Southern California. Currently, he is a faculty member at the University of Pennsylvania's School of Veterinary Medicine. 


\title{
AKA-TPG: A Program for Kinetic and Epidemiological Analysis of Data from Labeled Glucose Investigations Using the Two-Pool Model and Database Technology
}

\author{
RAYMOND C. BOSTON, Ph.D., ${ }^{1}$ DARKO STEFANOVSKI, M.Sc., ${ }^{2}$ \\ JAN E. HENRIKSEN, M.D., Ph.D., ${ }^{3}$ GLEN M. WARD, MD., Ph.D., ${ }^{4}$ \\ and PETER J. MOATE, M.Agr.Sc. ${ }^{1}$
}

\begin{abstract}
Background: The Two-Pool Glucose (TPG) model has an important role to play in diabetes research since it enables analysis of data obtained from the frequently sampled labeled (hot) glucose tolerance test (FSHGT). TPG modeling allows determination of the separate effects of insulin on the disposal of glucose and on the hepatic production of glucose. It therefore provides a basis for the accurate estimation of glucose effectiveness, insulin sensitivity, and the profile of the rate of endogenous glucose production. Until now, there has been no program available dedicated to the TPG model, and a number of technical reasons have deterred researchers from performing TPG analysis.

Methods and Results: In this paper, we describe AKA-TPG, a new program that combines automatic kinetic analysis of the TPG model data with database technologies. AKA-TPG enables researchers who have no expertise in modeling to quickly fit the TPG model to individual FSHGT data sets consisting of plasma concentrations of unlabeled glucose, labeled glucose, and insulin. Most importantly, because the entire process is automated, parameters are almost always identified, and parameter estimates are accurate and reproducible. AKA-TPG enables the demographic data of hundreds of individual subjects, their individual unlabeled and labeled glucose and insulin data, and each subject's parameters and indices derived from AKA-TPG to be securely stored in, and retrieved from, a database. We describe how the stratification and population analysis tools in AKA-TPG are used and present population estimates of TPG model parameters for young, healthy (without diabetes) Nordic men.

Conclusion: Researchers now have a practical tool to enable kinetic and epidemiological analysis of TPG data sets.
\end{abstract}

\section{INTRODUCTION}

W ORLDWIDE, METABOLIC SYNDROME is increasing in epidemic proportions, generating the need for increasingly sophisticated software to help with the elucidation of its pathogenesis. ${ }^{1,2}$

In 1979, Bergman and colleagues developed the glucose minimal model to be used to analyze data from the in vivo glucose tolerance test

\footnotetext{
${ }^{1}$ School of Veterinary Medicine, University of Pennsylvania, Kennett Square, Pennsylvania.

${ }^{2}$ Keck School of Medicine, University of Southern California, Los Angeles, California.

${ }^{3}$ Odense University Hospital, Department of Endocrinology, Diabetes Research Centre, Odense, Denmark.

${ }^{4}$ St. Vincent's Hospital, Melbourne, Australia.
} 
in order to obtain indices of the mechanisms that control the circulating levels of blood glucose. $^{3}$ These indices include insulin sensitivity $\left(S_{\mathrm{I}}\right)$ and glucose effectiveness $(S g)$. $S_{\mathrm{I}}$ represents the combined ability of insulin to promote the disposal of glucose and to inhibit the endogenous production of glucose. $S g$ represents the capacity of glucose to mediate its own disposal. In 1986, Bergman and Pacini ${ }^{4}$ released their stand-alone computer program (MinMod) providing clinical investigators a practical tool to facilitate Minimal Model analysis. Recently, Boston $^{5}$ and Boston et al. ${ }^{6}$ have released MinMod Millennium and AKA-Glucose, two updated versions of Minimal model software. An important feature of MinMod Millennium is that it employs an automated fitting algorithm that ensure good fits of the Minimal Model to almost every frequently sampled intravenous glucose test (FSIGT) data set. The distinguishing feature of AKA-Glucose is not only that it allows automatic fitting of FSIGT data, but that it couples this with database technologies.

In the mid-1980s, researchers, cognizant of deficiencies with the conventional glucose minimal model, employed the principle of tracer:tracee indistinguishability, in an attempt to separate the effects of insulin on the disposal of glucose and the suppression of hepatic glucose production. ${ }^{7,8}$ Initially, Cobelli et al. ${ }^{7}$ in 1986 used two one-compartment models, one for the unlabeled glucose and one for the "hot" glucose (either radiolabeled or stable isotope), with the major difference between these two models being an absence of a means of endogenous production of the labeled glucose. In 1991, Caumo et al. ${ }^{9}$ pointed out that the time course of the endogenous glucose production predicted by the one-pool model failed to conform to experimental data and that, contrary to theory, there was a difference between corresponding parameters estimated from the labeled and unlabeled models. Subsequently, Caumo et al. ${ }^{8}$ in 1993 introduced their two-pool glucose (TPG) model in which the glucose distribution space was split between an accessible pool and an exchangeable pool. They used deconvolution to estimate the input function, i.e., the hepatic glucose production rate (EGP), and obtained an EGP profile that more closely matched the pattern anticipated from physio- logical considerations. In their approach, they used the "hot" glucose model (they employed a stable isotope) to provide the transfer function, and the endogenous glucose level (predicted using an approach described by Cobelli et al. ${ }^{10}$ in 1990) as the response function. In order to ensure parameter identifiability, Caumo et al. ${ }^{8}$ introduced the following constraints: (1) only the exchangeable pool was assumed to be insulin sensitive, (2) the basal glucose disposal rate of the exchangeable pool was set to be onethird of the basal disposal rate of the accessible pool, and (3) a zero-order removal of glucose from the accessible pool to tissues such as brain was set to $1 \mathrm{mg} / \mathrm{kg} / \mathrm{min}$.

In the period between 1991 and 1999, there were few efforts to refine or apply the TPG model. ${ }^{11,12}$ In the early 1990s, the authors, when working with the TPG model, found problems with parameter estimability, especially physiologically implausible negative estimates for $k_{\mathrm{p}}$ (the parameter defining the firstorder removal of glucose from the accessible pool). In 1999, Cobelli et al. ${ }^{13}$ addressed the issue of estimability by introducing a Bayesian approach, i.e., the use of priors, to the estimation of the first-order parameters describing the exchange of glucose between the accessible and exchangeable pools. While the Bayesian approach eases the problem of parameter estimation, taken to the extreme, and if applied without caution, this approach resists the chance to discover new properties of hitherto unexplored populations. Although Cobelli et al., ${ }^{13}$ using theoretical arguments, were able to show that all of the parameters of the TPG model should be identifiable, noisy data in the initial phase of the frequently sampled labeled (hot) glucose tolerance test (FSHGT) make parameter estimability problematic.

The most recent improvement to the TPG model was made in 2003 by Toffolo and Cobelli. ${ }^{14}$ They tied the zero-order disposal from the accessible pool to the total glucose disposal, thus preventing a negative value for this component of glucose disposal and improving parameter estimability. Appendix $1 \mathrm{~A}$ depicts a schematic of the TPG model. Appendix 1B provides a complete account of the equations describing the TPG model. Appendix $1 \mathrm{C}$ provides definitions and descriptions of the variables as- 
sociated with the TPG model, their corresponding WinSAAM constructs, and their units.

Unlike the Minimal Model, the TPG model has not been extensively used by investigators. Possible reasons for this include the increased complexity of the experimental protocol and the necessity of using either radioactive tracers (with attendant problems) or stable isotopes (which entails considerable costs associated with chemicals and analytical devices). However, for researchers needing to measure the two effects of insulin (promoting glucose disposal and inhibiting hepatic production of glucose), we consider the principal reason has been the unavailability of a program dedicated to the automatic fitting of the TPG model to data. SAAMII code for fitting the TPG model to data has been freely available on the Internet, but for all except expert users of SAAMII, this code is not easy to implement or to obtain consistently identified accurate and reproducible parameter estimates. TPG investigations typically generate a considerable number of individual patient data sets (patient demographics and TPG data), which can create problems in terms of reliable storage and retrieval of individual files. Furthermore, there is a requirement that publicly funded experimental data must be retained for a period up to 10 years, in a form that can be readily accessible and retrievable. We address all of these problems by presenting a new computer program, AKA-TPG, an implementation of refinement of Toffolo and Cobelli ${ }^{14}$ to the TPG model coupled with database technology.

\section{RESULTS}

\section{Program description}

Since AKA-TPG incorporates many of the functions present in MINMOD Millennium and in AKA-Glucose, we, as far as possible, utilized the successful design and structural features present in these programs. ${ }^{5,6}$ As in MINMOD Millennium and AKA-Glucose, we utilized SAAM technology for the numerical integrator and processing kernel and an automated fitting algorithm. ${ }^{15}$ AKA-TPG is similar to AKA-Glucose in that it incorporates database technology and the powerful SAAM population modeling tool ${ }^{15}$ and the Microsoft (Redmond, WA) Jet version 4.0 database engine. The user can also use Microsoft Access 2000 or higher to independently access the database files that are populated by AKA-TPG. By orchestrating the interchange of data between these various programs, the AKA-TPG interface enables data to be easily imported into the program and analyzed, the data and results of analyses to be efficiently stored and retrieved, and the results of analyses (e.g., charts and reports) to be easily exported to a number of other productivity settings (Word, PowerPoint, Excel, STATA, WinSAAM). AKA-TPG is written in Visual Basic, and is designed to run on any PC with a Windows 98 or higher platform.

\section{The AKA-TPG interface}

Users of modern, scientific, software packages expect user-friendly interfaces with selfevident icons and features typical of those found in Windows-based programs. AKA-TPG has these types of features. The AKA-TPG interface is similar to that found in MinMod Millennium and AKA-Glucose, ensuring that users familiar with these programs would quickly become adept at using AKA-TPG. The main components of the AKA-TPG interface are six dialog windows: (1) Patient Data, (2) Observations, (3) Protocol, (4) Parameters, (5) Chart, and (6) Settings. The user gains access to each dialog window by selecting the appropriate window tab. In Figure 1, we see part of the Patient Data dialog window, which is where the user can add to the database or edit patient demographic data.

The Observations window (not shown) contains each subject's (sampling times, plasma concentrations of unlabeled glucose, labeled glucose, and insulin) and weighting structure in spreadsheet format. A new subject's data can be entered by selecting the "New" button. This produces a spreadsheet in which the new data can be manually entered, or the new data can be copied from an Excel spreadsheet and pasted into the spreadsheet. Alternatively, new data can be added to the database by selecting "Add." This results in a Windows file directory 


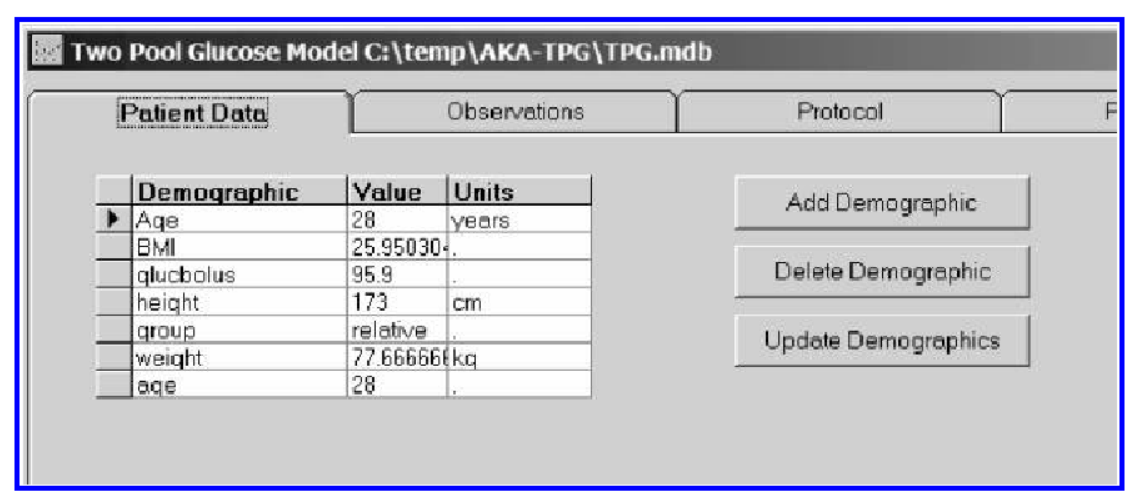

FIG. 1. Part of the Patient Data dialog window in AKA-TPG. The Patient Data tab is in bold font, indicating that the user has selected this tab to open this dialogue window. The text box contains the identifiers for the demographic features, their values, and corresponding units. The demographic features apply to every subject within the database, but the values of each demographic feature shown in the open dialog window pertain to a particular subject. Important demographic features include the patient's or subject's age, gender, body weight, ethnicity, etc. On the right side of the Patient Data dialog window are three Local Buttons that enable the user to add, delete, or edit the patient demographic features. BMI, body mass index.

from which the user can select a tab-delimited text file containing the new data. The current subject under focus can be deleted from the database by selecting the delete button.

The Protocol window (not shown) presents a schematic of the two-pool model and contains a text box in which the user can specify the administration protocols for insulin and labeled glucose.

\section{Fitting the model to data}

Prior to AKA-TPG, researchers faced with analyzing data from individual subjects and populations had no way to automatically analyze the data in a reproducible fashion to quickly obtain consistent, accurate, and precise estimates for TPG model parameters. The importance of the reproducibility of parameter estimates cannot be overstressed. This means that when a set of TPG data from a specific subject is analyzed, the parameter estimates should not depend upon the user or the time or place that the data are analyzed. Second, previous means of fitting the TPG model to data in SAAMII or WinSAAM were laborious and time consuming. Third, researchers needed a secure way to store the results of model fitting in a dedicated database. These aspects of TPG modeling are addressed in AKA-TPG. The fitting process is initiated in the Settings window. The user selects an appropriate local button to open an existing database or start a new database. The successful fitting of the TPG model to experimental data is greatly dependent upon good initial estimates of model parameters. To facilitate this process, we have included in the Settings window, as the default option, the population parameter means as reported by Toffolo and Cobelli $^{14}$ (Table 1), as initial estimates of major parameters. In order to allow for difficult cases or "nonhuman experimental subjects" the user can opt to enter, as initial estimates of model parameters, previously determined estimates of model parameters from specific populations.

Table 1. Default Initial Estimates and Their Standard Deviations of the Six Adjustable PARAMETERS IN THE TPG MODEL

\begin{tabular}{lcll}
\hline Parameter & Unit & Value & Standard deviation \\
\hline$k_{21}$ & $\min ^{-1}$ & 0.07 & 0.008 \\
$k_{12}$ & $\min ^{-1}$ & 0.08 & 0.014 \\
$k_{02}$ & $\min ^{-1}$ & 0.0039 & 0.00027 \\
$V_{1}$ & $\mathrm{dL} / \mathrm{kg}$ & 1.28 & 0.1 \\
$s_{k}$ & $\mathrm{~mL} \mu \mathrm{U}^{-1} \min ^{-1}$ & 0.00127 & 0.00028 \\
$p_{2}$ & $\min ^{-1}$ & 0.113 & 0.023 \\
\hline
\end{tabular}

These initial estimates of parameter values and their standard deviations are based on the findings of Toffolo and Cobelli. ${ }^{14}$ The automatic fitting algorithm in AKATPG uses these "priors" as default initial estimates of parameters so as to stabilize the estimation process and thereby improve parameter identifiability. The standard deviations of these parameters are employed as Bayesian constraints to counter problems with parameter estimability. The user can alter in AKA-TPG these initial estimates and Bayesian constraints if there is good justification. 


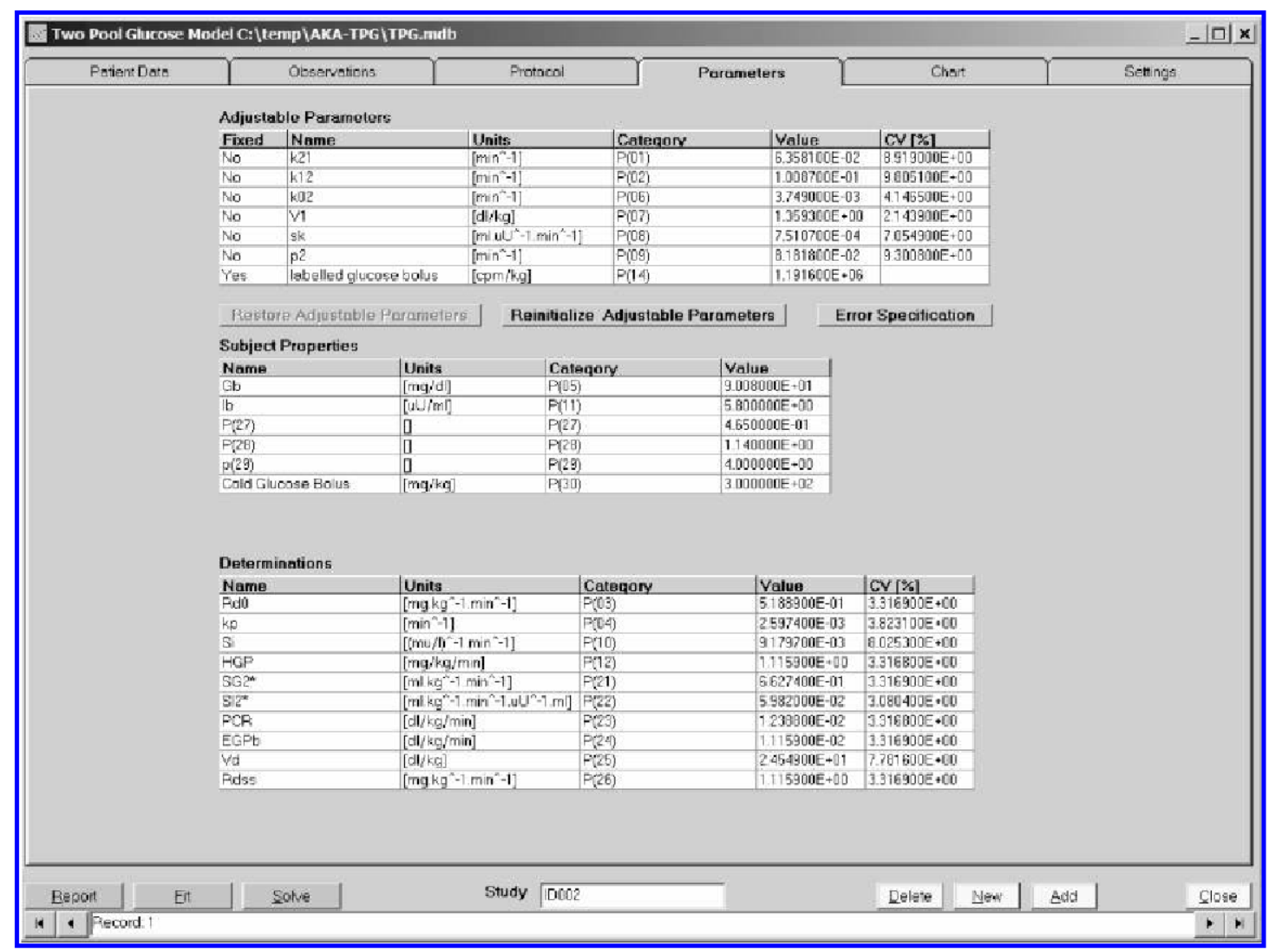

FIG. 2. The AKA-TPG Parameters dialog window. The names, units, best fit estimates, and uncertainties of the six adjustable parameters and 10 derived parameters of the TPG model are displayed in the Parameters window. Within the Parameters window are three local buttons-Restore Adjustable Parameters, Reinitialize Adjustable Parameters, and Error Specification - that are used to facilitate the model fitting process. Below the open dialogue window, one can see seven general buttons that initiate the major functions that can be carried out in AKA-TPG. These functions are: Report, Fit, Solve, Delete, New, Add, and Close. In between the "Solve" and "Delete" button is a small window showing the subject identifier (usually a name or unique identifying number). Below the subject identifier window is another small window containing the database record number corresponding to that particular subject. At the left and right on the bottom of the interface are arrows that allow the user to scroll between database records. Note that the Subject and Record windows show the subject and corresponding database record that is currently in "focus," i.e., retrieved from the database. CV, coefficient of variation; HGP, hepatic glucose production; PCR, plasma clearance rate.

The fitting process begins as described above, by the insertion in the text box in the Settings window of the initial estimates of the six adjustable parameters and five fixed parameters. To further enhance the likelihood that the model-fitting algorithm will locate feasible model solutions, we have employed the population parameter standard deviations reported by Toffolo and Cobelli ${ }^{14}$ as default Bayesian error constraints (see Table 1). In the Parameters window (Fig. 2), selecting the local button "Error Specification" results in a dropdown table containing the names of the adjustable parameters and their default Bayesian error constraints. A particularly useful at- tribute of AKA-TPG is that, after some experience of using AKA-TPG or in the light of a priori information, the user is able to appropriately edit these Bayesian error constraints in order to "best-fit" the model to a particular data set. At any time, the user can select the "Solve" button so as to invoke solution of the TPG model with the current parameter values. Selecting the "Fit" button automatically updates the parameters window with a "least-squares" best-fit of TPG model parameter estimates and their uncertainties and also updates the chart window with the new best-fit predictions of labeled glucose.

For each individual subject, model parame- 


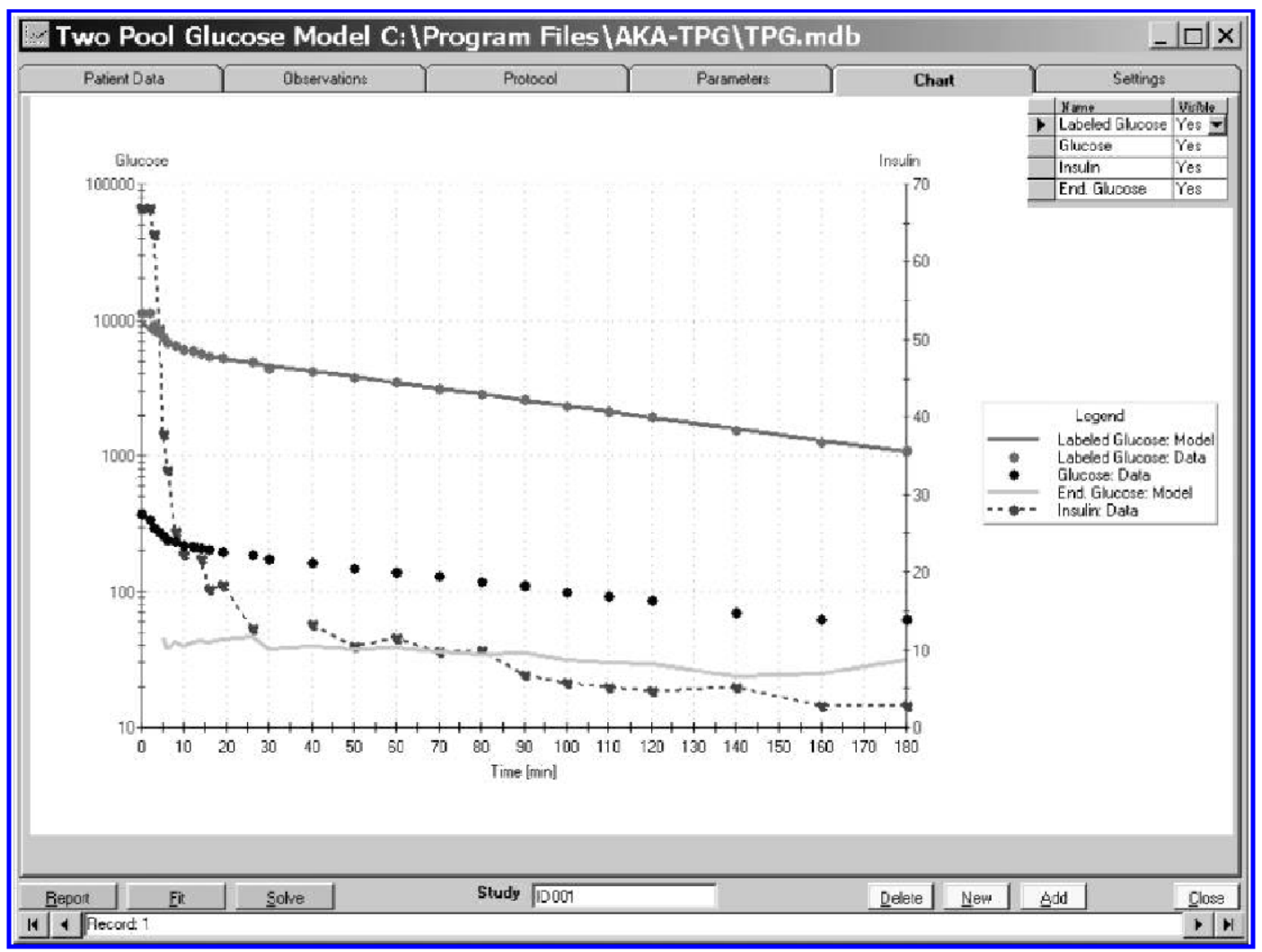

FIG. 3. The AKA-TPG Chart dialog window. AKA-TPG produces publication-quality charts in which the axis labels, axis type (logarithmic or linear), line style and symbol style can all be customized and color coded. The plasma data for the unlabeled glucose, labeled glucose, and insulin are depicted by dots while $I(t)$, the insulin driving function as well as AKA-TPG predictions of labeled glucose and endogenous glucose concentrations are depicted by lines (see chart legend).

ters are generally estimated with a coefficient of variation of less than $10 \%$. Using AKA-TPG, we have been able to successfully analyze data from a number of different projects in which the "hot" glucose involved both radiolabeled and stable isotope. In our experience, data from 30 subjects have been imported into AKA-TPG, the model has been fitted to the data, and reports on individual subjects and the population analysis report have all obtained within $30 \mathrm{~min}$.

\section{Obtaining results}

The entire purpose of TPG modeling is to obtain estimates of the parameters of the TPG model and to make predictions such as the instantaneous rate of endogenous glucose production. In AKA-TPG, we display in the Parameters window (Fig. 2) the names, units, best-fit estimates, and uncertainties of the six adjustable parameters and 10 derived parameters of the TPG model.
In the Chart window (see Fig. 3), we display a color-coded representation of the plasma unlabeled and labeled glucose and insulin. The TPG model predictions of labeled glucose and endogenous glucose production are shown by blue and green lines, respectively. Right clicking on the chart results in a dropdown menu that facilitates customization of the chart.

By selecting the "Report" button (bottom left in Fig. 3), the user activates a dropdown menu from which he or she can elect to print (1) a publication-quality chart depicting the time course of plasma concentrations unlabeled and labeled glucose and insulin in the current case under focus, (2) a tabulation of the current subject's model parameters and derived indices, together with their error estimates and units, (3) a table pertaining to the current case under focus, the time course of plasma concentrations of unlabeled glucose and labeled glucose, predicted unlabeled glucose, and predicted endogenous plasma glucose, and (4) a report for 
Table 2. The TPG Model: Parameter Nomenclature and Units, Parameter Description, WinSaAM Nomenclature, Population Mean Estimates, and

Their Standard Errors of TPG Parameters and Demographic Descriptors

\begin{tabular}{|c|c|c|c|c|}
\hline Parameter & Description & $\begin{array}{l}\text { WinSAAM } \\
\text { construct }\end{array}$ & Mean $\pm S E$ & Units $^{\mathrm{a}}$ \\
\hline$k_{21}$ & Rate constant & $\mathrm{P}(01)$ & $0.046 \pm 0.004$ & {$\left[\mathrm{~min}^{-1}\right]$} \\
\hline$k_{12}$ & Rate constant & $\mathrm{P}(02)$ & $0.046 \pm 0.006$ & {$\left[\min ^{-1}\right]$} \\
\hline$R d_{0}$ & $\begin{array}{l}\text { Insulin-dependent } \\
\text { glucose disposal }\end{array}$ & $\mathrm{P}(03)$ & $0.92 \pm 0.04$ & {$\left[\mathrm{mg} \mathrm{kg}^{-1} \mathrm{~min}^{-1}\right]$} \\
\hline$k_{\mathrm{p}}$ & Rate constant & $\mathrm{P}(04)$ & $0.0048 \pm 0.00024$ & {$\left[\min ^{-1}\right]$} \\
\hline$G_{\mathrm{b}}$ & Basal glucose & $\mathrm{P}(05)$ & $89 \pm 1.7$ & {$[\mathrm{mg} / \mathrm{dL}]$} \\
\hline$k_{02}$ & $\begin{array}{l}\text { Glucose disposal rate } \\
\text { from compartment } 2\end{array}$ & $\mathrm{P}(06)$ & $0.0044 \pm 0.00021$ & {$\left[\mathrm{~min}^{-1}\right]$} \\
\hline$V_{1}$ & Volume of accessible pool & $\mathrm{P}(07)$ & $1.4 \pm 0.06$ & {$[\mathrm{dL} / \mathrm{kg}]$} \\
\hline$s_{k}$ & Tissue insulin sensitivity & $\mathrm{P}(08)$ & $0.0016 \pm 0.00009$ & {$\left[\mathrm{~mL} \mu \mathrm{U}^{-1} \min ^{-1}\right]$} \\
\hline$p_{2}$ & A constant & $\mathrm{P}(09)$ & $0.060 \pm 0.0095$ & {$\left[\min ^{-1}\right]$} \\
\hline$S_{I}$ & Insulin sensitivity & $\mathrm{P}(10)$ & $0.042 \pm 0.0080$ & {$\left[\mathrm{~mL} \mu \mathrm{U}^{-1}\right]$} \\
\hline$I_{b}$ & Basal insulin & $\mathrm{P}(11)$ & $23.0 \pm 3.47$ & {$[\mu \mathrm{U} / \mathrm{mL}]$} \\
\hline HGP & Hepatic glucose production & $\mathrm{P}(12)$ & $1.97 \pm 0.090$ & {$[\mathrm{mg} / \mathrm{kg} / \mathrm{min}]$} \\
\hline Hot glucose bolus & Labeled glucose bolus & $\mathrm{P}(14)$ & & {$[\mathrm{mg} / \mathrm{kg} / 100]^{\mathrm{b}}$} \\
\hline$P C R$ & Plasma clearance rate & $P(23)$ & $0.022 \pm 0.0084$ & {$[\mathrm{dL} / \mathrm{kg} / \mathrm{min}]$} \\
\hline$E G P_{\mathrm{b}}$ & $\begin{array}{l}\text { Basal hepatic glucose } \\
\text { production }\end{array}$ & $\mathrm{P}(24)$ & $0.019 \pm 0.0009$ & {$\left[\mathrm{mg} \mathrm{kg}^{-1} \min ^{-1}\right]$} \\
\hline$R d_{s s}$ & Steady-state glucose disposal & $\mathrm{P}(26)$ & $1.97 \pm 0.09$ & {$\left[\mathrm{mg} \mathrm{kg}^{-1} \mathrm{~min}^{-1}\right]$} \\
\hline$q_{1}(0)$ & Dose of labeled glucose & $\mathrm{IC}(1)$ & & {$[\mathrm{mg} / \mathrm{kg} / 100]^{\mathrm{b}}$} \\
\hline$\alpha$ & Toffolo's $\alpha$ & $\mathrm{P}(27)$ & 0.465 & Dimensionless \\
\hline P28 & Toffolo's constant & $\mathrm{P}(28)$ & 1.14 & Dimensionless \\
\hline P29 & Cobelli's constant & $P(29)$ & 4 & Dimensionless \\
\hline$V_{\mathrm{d}}$ & Volume of distribution & $\mathrm{P}(25)$ & $2.3 \pm 0.09$ & {$[\mathrm{dL} / \mathrm{kg}]$} \\
\hline$S_{G}^{*}$ & $\begin{array}{l}\text { Glucose effectiveness } \\
\text { (two pool) }\end{array}$ & $\mathrm{P}(21)$ & $1.19 \pm 0.04$ & {$\left[\mathrm{~mL} \mathrm{~kg}{ }^{-1} \mathrm{~min}^{-1}\right]$} \\
\hline$S_{I}^{*}$ & $\begin{array}{l}\text { Insulin sensitivity } \\
\text { (two pool) }\end{array}$ & $\mathrm{P}(22)$ & $0.18 \pm 0.01$ & {$\left[\mathrm{~mL} / \mathrm{kg} / \min (\mu \mathrm{U} / \mathrm{mL})^{-1}\right]$} \\
\hline$D$ & Unlabeled glucose bolus & $\mathrm{IC}(4)$ & $300 \pm 2.6$ & {$[\mathrm{mg} / \mathrm{kg}]$} \\
\hline Age & Years & & $32.1 \pm 1.35$ & [years] \\
\hline$B M I$ & Body mass index & & $26.6 \pm 0.98$ & {$\left[\mathrm{~kg} \mathrm{~m}^{-2}\right]$} \\
\hline
\end{tabular}

The population estimates of TPG parameters were for 15 young, healthy(without diabetes) Nordic men involved in an investigation funded by the Danish Diabetes Association, the Clinical Research Institute, Odense University Hospital, the Novo Nordisk Foundation, and Aage Louis-Hansens Memorial Foundation.

aUnits for stable isotope dose.

${ }^{\mathrm{b}}$ For a radioactive label, replace $\mathrm{mg} / \mathrm{kg} / 100$ with $\mathrm{cpm} / \mathrm{kg}$.

all the studies in the database or (5) a WinSAAM file of the current subject.

\section{Population studies}

A typical investigation in which we employed AKA-TPG involved an analysis of genetic risk factors for diabetes in Nordic men. Data for all subjects in the investigation were entered into the AKA-TPG database and individually fitted as described above. A population analysis of the "control" group of 15 "young, healthy (without diabetes) men" was initiated by selecting the "Report Button" in the Settings window. This produced a dropdown menu from which the "Population Analysis" option was activated by means of a radio button. The constraints describing the control stratum were entered into a dialog box, and the population analysis was performed by selecting the "Analyze Stratum" button. This generated an extensive amount of useful output in 10 worksheets in spreadsheet format. In particular, the "Population Parameter" worksheet contains unbiased estimates of the population means of the six adjustable parameters of the labeled TPG model and their respective population fractional standard deviations. Table 2 lists nomenclature of the TPG model parameters, their units, description, WinSAAM nomenclature, and the population estimates and standard errors of the TPG parameters for 
"young, healthy (without diabetes) Nordic men."

In this investigation, the population mean estimates for TPG parameters were as follows: $V_{1}=1.4 \pm 0.06 \quad \mathrm{dL} / \mathrm{kg}, \quad k_{21}=0.046 \pm 0.004$ $\min ^{-1}, \quad k_{12}=0.046 \pm 0.006 \min ^{-1}, \quad k_{02}=$ $0.0044 \pm 0.0002 \mathrm{~min}^{-1}, \quad p_{2}=0.060 \pm 0.009$ $\min ^{-1}, s_{k}=0.0016 \pm 0.00009 \mathrm{~min}^{-1} \mu \mathrm{U}^{-1} \mathrm{~mL}$, $S_{\mathrm{G}}^{2^{*}}=1.19 \pm 0.04 \mathrm{~mL} \mathrm{~kg}{ }^{-1} \mathrm{~min}^{-1}$, and $S_{\mathrm{I}}^{2^{*}}=$ $0.18 \pm 0.01 \mathrm{~mL} / \mathrm{kg} / \mathrm{min}(\mu \mathrm{U} / \mathrm{mL})^{-1}$. These estimates for a "normal-healthy" population of "Nordic" men are somewhat different to the estimates reported by Toffollo and Cobelli ${ }^{14}$ for a mixed population of American and Italian subjects: $V_{1}=1.4 \pm 0.04 \mathrm{dL} / \mathrm{kg}, k_{21}=0.070 \pm$ $0.008 \mathrm{~min}^{-1}, k_{12}=0.080 \pm 0.01 \mathrm{~min}^{-1}, k_{02}=$ $0.004 \pm 0.0002 \mathrm{~min}^{-1}, p_{2}=0.12 \pm 0.02 \mathrm{~min}^{-1}$, $s_{k}=0.0012 \pm 0.0003 \mathrm{~min}^{-1} \mu \mathrm{U}^{-1} \mathrm{~mL}, S_{\mathrm{G}}^{2^{*}}=$ $0.85 \pm 0.14 \mathrm{~mL} \mathrm{~kg}^{-1} \mathrm{~min}^{-1}$, and $S_{\mathrm{I}}^{2^{*}}=0.14 \pm$ $0.03 \mathrm{~mL} / \mathrm{kg} / \mathrm{min}(\mu \mathrm{U} / \mathrm{mL})^{-1}$. The "normalhealthy" population studied by Toffolo and Cobelli $^{14}$ lived in different countries (the United States and Italy) and may very well have differed in other key (unspecified) demographic features (e.g., diet) compared with the "normal-healthy" population in the current investigation. Yet, similarities in the magnitudes of the major health-related TPG parameters $\left(S_{\mathrm{G}}^{2^{*}}\right.$ and $\left.S_{\mathrm{I}}^{2^{*}}\right)$, especially those scaled by body weight, suggest that these parameters do in fact define a "normal-healthy" population, and this supports the utility of the TPG modeling approach. Differences in the magnitudes of the "mixing" parameters, especially $k_{12}, k_{21}$, and $p_{2}$ (the parameter describing the rate of decline of insulin action in a remote compartment) are intriguing and warrant further research.

\section{CONCLUSIONS}

In this paper we have described AKA-TPG, the first software specifically developed for fitting the TPG model to data. AKA-TPG has a user-friendly interface, and it allows the user to automatically and quickly obtain accurate, precise, and reproducible estimates of all TPG parameters and indices for individual subjects. The population tool within AKA-TPG makes it possible for investigators and epidemiologists to determine mean TPG parameter estimates for populations and strata within populations. The database technology within AKA-TPG allows the user to store and retrieve from a secure database all TPG-related data from many hundreds of subjects.

\section{REFERENCES}

1. Centers for Disease Control and Prevention: New 2003 Diabetes Data. Atlanta: Centers for Disease Control and Prevention, 2003.

2. Zimmet P, Alberti KG, Shaw J: Global and societal implications of the diabetes epidemic. Nature 2001; 414:782-787.

3. Bergman RN, Ider YZ, Bowden CR, Cobelli C: Quantitative estimation of insulin sensitivity. Am I Physiol 1979;236:E667-E677.

4. Pacini G, Bergman RN: MINMOD-a computer program to calculate insulin sensitivity and pancreatic responsivity from the frequently sampled intravenous glucose tolerance test. Comput Methods Programs Biomed 1986;23:113-122.

5. Boston RC, Stefanovski D, Moate PJ, Sumner AE, Watanabe RM, Bergman RN: MINMOD Millennium: a computer program to calculate glucose effectiveness and insulin sensitivity from the frequently sampled intravenous glucose tolerance test. Diabetes Technol Ther 2003;5:1003-1015.

6. Boston RC, Moate PJ, Stefanovski D, Sumner AE, Bergman RN: AKA-Glucose: a program for kinetic and epidemiological analysis of frequently sampled intravenous glucose tolerance test data using database technology. Diabetes Technol Ther 2005;7:298-307.

7. Cobelli C, Pacini G, Toffolo G, Sacca L: Estimation of insulin sensitivity and glucose clearance from minimal model: new insights from labelled IVGTT. Am I Physiol Endocrinol Metab 1986;250:E591-E598.

8. Caumo A, Cobelli C: Hepatic glucose production during labelled IVGTT: estimation by deconvolution with a new minimal model. Am J Physiol Endocrinol Metab 1993;264:E829-E841.

9. Caumo A, Giacca A, Morgese G, Pozza G, Micossi P, Cobelli C: Minimal models of glucose disappearance: lessons from the labelled IVGTT. Diabet Med 1991;8:822-832.

10. Cobelli C, Toffolo G: Constant specific activity input allows reconstruction of endogenous glucose concentration in non-steady state. Am J Physiol Endocrinol Metab 1990;258:E1037-E1040.

11. Ward GM, Weber KM, Walters IM, Aitken PM, Lee B, Best JD, Boston RC, Alford FP: A modified minimal model analysis of insulin sensitivity and glucosemediated glucose disposal in insulin-dependent diabetes. Metabolism 1991;40:4-9.

12. Overkamp D, Gautier D, Renn W, Pickert A, Scheen AJ, Schmulling RM, Eggstein M, Lefebvre PJ: Glucose turnover in humans in the basal state and after intravenous glucose: a comparison of two models. Am J Physiol Endocrinol Metab 1997;273:E284-E296. 
13. Cobelli C, Caumo A, Omnetto M: Minimal model Sg overestimation and SI underestimation: improved accuracy by Bayesian two-compartmental model. Am J Physiol Endocrinol Metab 1999;277:E481-E488.

14. Toffolo G, Cobelli C: The hot IVGTT two compartmental minimal model: an improved version. Am J Physiol Endocrinol Metab 2003;284:E317-E321.

15. Stefanovski D, Moate PJ, Boston RC: WinSAAM: a Windows-based compartmental modeling system. Metabolism 2003;52:1153-1166.
Address reprint requests to: Raymond C. Boston, Ph.D. University of Pennsylvania New Bolton Centre 382 West Street Road Kennett Square, PA 19348

E-mail: drrayboston@yahoo.com

APPENDIX 1A. A SCHEMATIC OF THE TPG MODEL

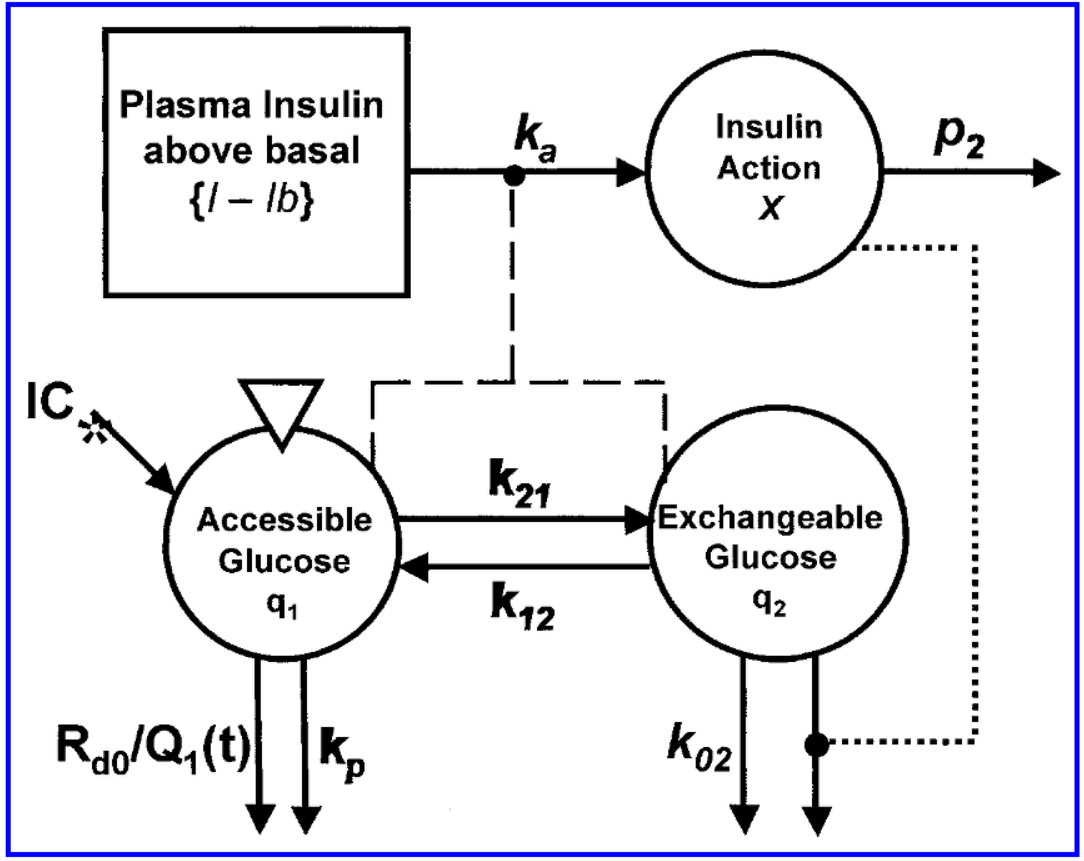

Note, in this schematic, $k_{a}=p_{2} s_{k}$.

\section{APPENDIX 1B. TPG MODEL EQUATIONS}

$$
\begin{aligned}
& q_{1}(t)^{\prime}=-\left(k_{\mathrm{p}}+\frac{R d_{\mathrm{o}}}{Q_{1}(t)}+k_{21}\right) * q_{1}(t)+k_{12} * q_{2}(t) \\
& q_{1}(0)=d \\
& q_{2}(t)^{\prime}=k_{21} * q_{1}(t)-\left(k_{02}+x(t)+k_{12}\right) * q_{2}(t) \\
& x(t)^{\prime}=-p_{2}\left[x(t)-s_{k}\left\{I(t)-I_{\mathrm{b}}\right\}\right] \\
& I(t)-I_{\mathrm{b}}=0 \text { if } I(t) \leq I_{\mathrm{b}} \quad x(0)=0 \\
& g_{1}(t)=\frac{q_{1}(t)}{V_{1}}
\end{aligned}
$$


$G_{1}(t)=\frac{Q_{1}(t)}{V_{1}}$

$G_{\mathrm{e}}(t)=G_{1}(t)-\left(\frac{G_{0} * q_{1}(t)}{q_{1}(0)}\right)$

$\Delta G_{\mathrm{E}}(t)=\int_{0}^{t} g_{1}(t, T) * \Delta E G P(T) * d T$

$k_{\mathrm{p}}=\left(\frac{1.14 * k_{21} * k_{02}}{k_{02}+k_{12}}\right)$

$k_{\mathrm{p}}+\frac{R d_{0}}{V_{1} G_{\mathrm{b}}}=\frac{3 k_{21} k_{02}}{k_{02}+k_{12}}$

$P C R=\left(\frac{4 * V_{1} * k_{21} * k_{02}}{k_{02}+k_{12}}\right)$

$E G P_{\mathrm{b}}=P C R * G_{\mathrm{b}}$

$S_{\mathrm{G}}^{2 *}=100 * V_{1} *\left(k_{\mathrm{p}}+\frac{k_{21} * k_{02}}{k_{02}+k_{12}}\right)$

$S_{\mathrm{I}}^{2^{*}}=\left(\frac{100 * s_{k}^{*} k_{21} * k_{12} * V_{1}}{\left(k_{02}+k_{12}\right)^{2}}\right)$

PCR, plasma clearance rate.

APPENDIX 1C. DEFINITIONS OF VARIABLES IN THE TPG MODEL

\begin{tabular}{llcc}
\hline Variables & \multicolumn{1}{c}{ Description } & $\begin{array}{c}\text { WinSAAM } \\
\text { construct }\end{array}$ & \multicolumn{1}{c}{ Units $^{\mathrm{a}}$} \\
\hline$q_{1}(t)$ & Accessible pool labeled glucose & $\mathrm{F}(1)$ & {$[\mathrm{mg} / \mathrm{kg}]^{\mathrm{b}}$} \\
$q_{2}(t)$ & Exchangeable pool labeled glucose & $\mathrm{F}(2)$ & {$[\mathrm{mg} / \mathrm{kg}]^{\mathrm{b}}$} \\
$x(t)$ & Insulin action & $\mathrm{F}(3)$ & {$[\mathrm{min}-1]$} \\
$G_{1}(t)$ & Unlabeled glucose concentration & $\mathrm{FF}(4)$ & {$[\mathrm{mg} / \mathrm{dL}]$} \\
$G_{e}(t)$ & Endogenous glucose & $\mathrm{G}(10)$ & {$[\mathrm{mg} / \mathrm{dL}]$} \\
$I(t)$ & Insulin concentration & $\mathrm{FF}(5)$ & {$[\mathrm{uU} / \mathrm{mL}]$} \\
$q_{1}(t)^{\prime}$ & Rate of change of accessible labeled & $\mathrm{UF}(1)$ & {$[\mathrm{mg} / \mathrm{kg} / \mathrm{min}]^{\mathrm{b}}$} \\
& $\quad$ glucose pool & $\mathrm{UF}(2)$ & {$[\mathrm{mg} / \mathrm{kg} / \mathrm{min}]^{\mathrm{b}}$} \\
$q_{2}(t)^{\prime}$ & $\begin{array}{l}\text { Rate of change of exchangeable labeled } \\
\text { glucose pool }\end{array}$ & $\mathrm{UF}(3)$ & {$\left[\mathrm{min}^{-2}\right]$} \\
$x(t)^{\prime}$ & Rate of change of insulin action &
\end{tabular}

aUnits for stable isotope dose.

${ }^{b}$ For radioactive label, replace $\mathrm{mg}$ with $\mathrm{cpm}$. 
This article has been cited by:

1. 2007. Current literature in diabetes. Diabetes/Metabolism Research and Reviews 23:10.1002/dmrr.v23:7, i-ix. [CrossRef] 